\title{
The unbearable heaviness of reductionism: the paradigm of the antiatherogenic diet
}

\author{
Marco Bobbio, ${ }^{1}$ Antonio Bonaldi, ${ }^{2}$ Luigi Lusiani, ${ }^{3}$ Emma Kidd ${ }^{4}$ \\ ${ }^{1}$ Slow Medicine General Secretariat, Former Director Cardiovascular Department, Santa Croce e Carle Hospital, Cuneo, Italy; \\ ${ }^{2}$ Slow Medicine President, former Medical Director, Brescia, Italy; ${ }^{3}$ Department Internal Medicine, Hospital of Castelfranco \\ Veneto (TV), Italy; ${ }^{4}$ Schumacher College, Dartington, Totnes, UK
}

\begin{abstract}
Reducing a complex phenomenon into a simplified description may help us to understand general issues and to make limited decisions, but can hardly allow us to accurately predict the consequences of an intervention. The case of the antiatherogenic diet is discussed. Simplified approaches, aimed at correcting single components of a diet, privileging the lipid theory over more comprehensive ones, sustained by the illusion that removing one single substance (the lowering-cholesterol mantra) would defeat cardiovascular disease, have led us to faulty results, because the interactions among components and their interconnections are not negligible. The industry's interest in shifting the diet of millions of people in well-defined and profitable directions (lowfat, but sugar-rich for tastefulness reasons, as an example), in advance of well founded scientific evidence about benefit, has been mostly relevant. Nowadays, it is well recognized that refined and sweetened foods, not fats, are the main cause of obesity and diabetes, and that the cardiovascular risk is more dependent on the quantity of food consumed rather than the type of food. In recent years the attention of the researchers has moved from qualitative dietary studies and unnatural regimes, to quantitative diets and more naturally balanced regimes, within the concept of globally health life styles, against any reductionism.
\end{abstract}

\section{Introduction}

Simplifying difficult issues is an essential part of life; reducing a complex phenomenon into a simplified description helps us to understand the general ways in which this phenomenon functions. The reduction enables us to make predictions and makes it possible for us to intervene in straightforward ways when problems arise. For example, if a watch stops, I can take it apart, analyze the individual gears, find the cause of the malfunction and adjust or change a single piece, according to a linear logic of cause and effect. On the other hand, when the systemic elements of a phenomenon are linked by an intricate network of relationships (often

Correspondence: Luigi Lusiani, Internal Medicine, Hospital of Castelfranco Veneto, via dei Carpani 16/Z, 31033 Castelfranco Veneto (TV), Italy.

E-mail: luigi.lusiani@aulss2.veneto.it

Key words: Cardiovascular risk; antiatherogenic diet.

Received for publication: 4 May 2017

Accepted for publication: 5 May 2017.

Conflict of interest: the authors declare no potential conflict of interest.

This work is licensed under a Creative Commons Attribution NonCommercial 4.0 License (CC BY-NC 4.0).

CCopyright M. Bobbio et al., 2017

Licensee PAGEPress, Italy

Italian Journal of Medicine 2017; 11:322-324

doi:10.4081/itjm.2017.869 indecipherable) the process of simplification becomes a much more delicate task; the results of which can make it difficult to accurately predict the consequences of our interventions. To illustrate this let's consider the introduction of a selective herbicide, which, in theory, should only destroy specific weeds species. However, if this herbicide also prevents the replication of certain insects, and these insects are the primary source of food for a particular species of bird, a likely unintended consequence is that this bird species will become extinct. Similarly, through a chain of interconnected and unpredictable events, instead of predicted benefits, the elimination of a single element from the human diet can create damaging unintended consequences; banishing fats and cholesterol for half a century from the diet of Western populations is one such example.

\section{The mantra}

The concept that fat and cholesterol intake should be lowered to reduce the risk of heart attacks has dominated dietary advice for five decades. Post-war, two diverse theories arose; John Yudkin's theory considered sugar to be the major cause of coronary heart disease, whilst Ancel Key's theory suggested that the major cause was dietary fat, especially saturated fat and cholesterol. Keys' theory was based on a comparison between the rate of cholesterol in the blood and the rate of heart failure in the populations of 7 countries. ${ }^{1}$ However, in this highlyquoted investigation, the analysis of Crete's population was based on data from only 9 people. Despite this, Keys' theory prevailed, supported by numerous other 
studies. The data from these studies included the high presence of cholesterol in atherosclerotic plaques; the occurrence of arterial plaques in rabbits fed with highfat diets; the increased incidence of cardiovascular events in hypercholesterolemic patients; the differences of infarction rates between populations with different types of diet; and, lastly, the fact that the use of cholesterol-lowering drugs, such as statins, had reduced the incidence of cardiovascular events and death. ${ }^{2}$ Thus, cholesterol was considered, for almost half a century, the leading killer of modern times and the low-fat diet became the cornerstone of nutrition in the Western world.

\section{Cholesterol killer}

In 1980 the collection of these data led the US Department of Health and Human Services to define rules (Dietary Guidelines for Americans) for a diet that would reduce the burden of cardiovascular events based on two dietary changes; reducing fat consumption below $30 \%$ of the total dietary intake of calories and reducing saturated fatty acids below $10 \%$. These recommendations would also serve to avoid weight gain, since fats have a higher content of calories ( 9 per gram) than carbohydrates ( 4 per gram). Soon after relentless campaigns were launched which promoted the reduction of fat percentages in the mean American diet from 40 to $30 \%$. However, instead of the predicted benefits, these dietary changes caused some unexpected and paradoxical consequences - increased incidence of type 2 diabetes, the prevalence of obesity was tripled and a halt occurred in the progressive reduction of cardiovascular disease, despite the use of pharmacological and interventional therapies. ${ }^{3,4}$ Meanwhile, the food industry, under the pressure of huge economic interests, contributed to this trend, suggesting the replacement of butter (animal origin, rich in saturated fatty acids and solid at room temperature) with substitutes such as margarine (vegetable origin, rich in unsaturated fatty acids and liquid at room temperature), neglecting the fact that the process of solidification of unsaturated fatty acids involves their hydrogenation - a transformation into saturated fatty acids - and compensated for the lowered palatability of these processed foods with the addition of sugar. Once butter had been replaced in industrial food preparation and home cooking, it was then discovered that these new products actually increased low-density lipoprotein (LDL) cholesterol, reduced high-density lipoprotein cholesterol and increased the incidence of cardiovascular events. ${ }^{5}$ That is why the Food and Drug Administration (FDA) banned margarines in 2014.

\section{Much research, many uncertainties}

During the last half-century, an enormous amount of data from observational and experimental research has been accumulated on the relationship between food and health; yet it suffers from specific limitations. The observational studies were based on one-off questionnaires investigating people's usual diets; this means that self-declarations are not always verifiable (the National Health and Nutrition Examination Survey found that daily calories introduced were incompatible with life in $2 / 3$ of the spontaneous statements of the respondents ${ }^{6}$ ), secondly, that reducing each diet into its individual components (carbohydrates, lipids, proteins, salts, vitamins, etc.) introduces distortions and, thirdly, that over the years (or even the seasons) there are changes in dietary habits not accounted for in the questionnaire. In the case of experimental studies, having to force large populations to adapt to a very particular diet means that research of this type cannot be conducted for long periods of time, and therefore these studies can only be used to evaluate surrogate end points (modification of biological parameters, rather than clinical outcomes).

Despite these biases, which affected the results of research in support of both the lipid theory and glucose theory, more credit was given to the first hypothesis the result of which was a worldwide mass experiment, uncontrolled, based on weak data and influenced by conflicts of interest.

\section{The role of industry}

Changing the diets of millions of people can move huge economic resources, therefore, it was clear that the food industry would profit from influencing eating behavior. Kearn, ${ }^{7}$ for example, restored the role played by the Sugar Research Foundation. In 1954, observing the growing interest in low-fat diets, the Foundation assumed a strategic market opportunity and began to fund researchers to demonstrate the benefits of a diet rich in sugars. They enlisted opinion leaders to support this hypothesis and then started information campaigns to demonstrate that sugar is essential for life.

\section{The turning point}

With the passing of years and the accumulation of scientific evidence favoring the sugar hypothesis, dominant thought has finally cracked and the alternative hypothesis has forged ahead, so much so that the scientific community now believes that there is not, in fact, such a close relationship between cholesterol intake, the concentration of serum cholesterol and cardiovascular events. ${ }^{89}$ Furthermore, for years now physicians have been recommending that their patients reduce fat intake, yet finding that LDL cholesterol levels were only reduced minimally in the face of substantial dietary changes. However, on recognizing that refined and sweetened foods, not fats, are the main cause of obesity and diabetes, the eighth edition of the Dietary Guidelines for Americans ${ }^{10}$ in 2015 stated that food-related cholesterol intake should no longer be considered a risk factor for heart disease, and that the limitation on the 
percentage of fat being consumed in a diet should be abolished, changing two basic concepts of the dominant recommendations. The guidelines concluded that, to reduce the total fat (replacing it with carbohydrates) does not reduce the risk of cardiovascular events.

\section{Learning from mistakes}

For many years, food science has limited itself to studying the metabolic activity of individual components ${ }^{11}$ (and still does); neglecting to study the interactions between components, and disregarding the network of interconnections that link them. This reductionist approach fuelled the myth of an ideal diet that is built only on substances deemed useful, ${ }^{12}$ and, by ignoring all contrary data, imposed a single and irrefutable thought; the illusion that removing one substance from the diet of millions of people would defeat cardiovascular disease.

What follows are some considerations that have been neglected in this approach: i) the lipid hypothesis is evocative but simplistic; ii) biology does not work in a linear fashion, but as a complex system; iii) the body's mechanisms that regulate the welfare and the development of disease do not depend only on energy intake; iv) metabolism is not simply regulated by individual elements, but by the combination of ingredients, by the rhythms of meals, by habits, by cycles of nature, by local ingredients and finally by the pleasure of eating; v) the elimination of an important component in the diet implies the replacement with something else; vi) the food industry interferes with science in ways which directly affect research and consumer choices, introducing new products even before they have been proven safe; vii) a comprehensive healthy life (no smoking, weight control, regular exercise and a careful diet with the adoption of vegetables, fruit, fish and unrefined carbohydrates) can halve the relative risk of cardiovascular events even in people at high genetic risk. ${ }^{13}$

In fact, in recent years the attention of researchers has shifted from qualitative dietary studies, which often impose unnatural dietary regimes, to quantitative diets, given that obesity and cardiovascular risk are more dependent on the quantity of food consumed, rather than the type of food. ${ }^{14}$

After decades of propaganda, how can we now remove a concept that is so ingrained? People continue to avoid dairy products, or at most base their choices on partially or wholly skimmed milk products (without taking into account - for example - the impact of the reduction of calcium on osteoporosis and spontaneous fractures). A 2004 survey found that the majority of Americans avoided foods containing fats and cholesterol, and consumed excessive amounts of refined carbohydrates (white bread, rice, crackers, cereals, baked goods) as their main source of calories. How long will it take to change and transform these deeply rooted habits? As Ioannidis argues: ${ }^{15}$ Definitive solutions will not come from another million observational papers or a few small randomized trials. Randomized trials are needed mainly to inform the design of pivotal mega-trials of comprehensive interventions. We should also continue to explore other aspects of food and nutrition such as food security, sustainability, social inequalities, famine, and impact of food production on climate change - that may also affect human societies and wellbeing through multiple pathways. Food and nutrition may well make a major difference, but perhaps for reasons other than those that are usually touted, debated about, and contradicted.

\section{References}

1. Keys A. Coronary heart disease in seven countries. Circulation 1970;41:1-200.

2. Scandinavian Simvastatin Survival Study Group. Randomised trial of cholesterol lowering in 4444 patients with coronary artery disease: the Scandinavian Simvastatin Survival Study (4S). Lancet 1994;344:1383-9.

3. Mansoor N, Vinknes KJ, Veierød MB, Retterstøl K. Effects of low-carbohydrate diets v. low-fat diets on body weight and cardiovascular risk factors: a meta-analysis of randomised controlled trials. Br J Nutr 2016;115:466-79.

4. Ludwig DS, Friedman MI. Increasing adiposity: consequence or cause of overeating? JAMA 2014;311:2167-8.

5. Mensink RP, Katan MB. Effect of dietary trans fatty acids on high-density and low-density lipoprotein cholesterol levels in healthy subjects. N Engl J Med 1990;323:439-45.

6. Archer E, Hand GA, Blair SN. Validity of US nutritional surveillance: National Health and Nutrition Examination Survey caloric energy intake data, 1971-2010. PLoS One 2013;8:e76632.

7. Kearns CE, Schmidt LA, Glantz SA. Sugar industry and coronary heart disease research. A historical analysis of internal industry documents. JAMA Intern Med 2016; 176:1680-5.

8. Mozaffarian D, Ludwig DS. The 2015 US Dietary Guidelines lifting the ban on yotal dietary fat. JAMA 2015;313:2421-2.

9. Kromhout D. Where the latest US dietary guidelines are heading. BMJ 2015;351:h4034.

10. US Department of Health and Human Services; US Department of Agriculture. 2015-2020 Dietary Guidelines for Americans. 8th ed. Washington, DC: US Dept of Health and Human Services; December 2015. Available from: http://www.health.gov/DietaryGuidelines

11. Satija A, Yu E, Willett WC, Hu FB. Understanding nutritional epidemiology and its role in policy. Adv Nutr 2015;6:5-18.

12. Ludwig DS. Lowering the bar on the low-fat diet. JAMA 2016;316:2087-8.

13. Khera AV, Emdin CA, Drake I et al. Genetic risk, adherence to a healthy life style, and coronary disease. N Engl J Med 2016;375:2349-58.

14. Veerman JL. Dietary fats: a new look at old data challenges established wisdom. BMJ 2016;353:11512.

15. Ioannidis JPA. Implausible results in human nutrition research. BMJ 2013;347:f6698. 